\title{
Venetoclax with azacitidine targets refractory MDS but spares healthy hematopoiesis at tailored dose
}

\author{
Stefanie Jilg ${ }^{{ }^{*}+} @$, Richard T. Hauch ${ }^{1 \dagger}$, Johanna Kauschinger ${ }^{1}$, Lars Buschhorn ${ }^{1}$, Timo O. Odinius ${ }^{1}$, Veronika Dill ${ }^{1}$, \\ Catharina Müller-Thomas ${ }^{1}$, Tobias Herold ${ }^{2}$, Peter M. Prodinger ${ }^{3}$, Burkhard Schmidtt ${ }^{4}$. Dirk Hempel ${ }^{5}$, \\ Florian Bassermann ${ }^{1,6}$, Christian Peschel ${ }^{1,6}$, Katharina S. Götze ${ }^{1,6}$, Ulrike Höckendorf ${ }^{1}$, Torsten Haferlach ${ }^{7}$ \\ and Philipp J. Jost ${ }^{1,6^{*}}$
}

\begin{abstract}
Patients with Myelodysplastic Syndromes (MDS) and secondary Acute Myeloid Leukemia (SAML) have a very poor prognosis after failure of hypomethylating agents (HMA). Stem cell transplantation is the only effective salvage therapy, for which only a limited number of patients are eligible due to age and comorbidity. Combination therapy of venetoclax and azacitidine (5-AZA) seems to be a promising approach in myeloid malignancies, but data from patients with HMA failure are lacking. Furthermore, a considerable concern of combination regimens in elderly AML and MDS patients is the toxicity on the remaining healthy hematopoiesis. Here, we report in vitro data showing the impact of venetoclax and 5-AZA, alone or in combination, in a larger cohort of MDS/sAML patients $(n=21)$, even after HMA failure $(n=13)$. We especially focused on the effects on healthy hematopoiesis and the impact on colony forming capacity as a parameter for long-term effects. To the best of our knowledge, we show for the first time that venetoclax in combination with capped dose of 5-AZA targets cell malignancies, while sparing healthy hematopoiesis.
\end{abstract}

Keywords: MDS, Venetoclax, Combination therapy, Hematotoxicity, HMA failure

Patients with Myelodysplastic Syndromes (MDS) and secondary Acute Myeloid Leukemia (sAML) have a very poor prognosis after failure of hypomethylating agents (HMA). For these patients, stem cell transplantation represents the only effective salvage therapy, for which only a limited number of patients are eligible due to age and comorbidity. Further durable treatment options are completely lacking at the moment [1].

A Phase $1 \mathrm{~b}$ multicentre study (M15-522) has been initiated to determine the safety of venetoclax treatment, as monotherapy or in combination with 5 -azacitidine (5-AZA), in subjects with relapsed/refractory MDS. This

\footnotetext{
*Correspondence: stefanie.jilg@tum.de; philipp.jost@tum.de

${ }^{\dagger}$ Stefanie Jilg and Richard T. Hauch contributed equally to this work

${ }^{1}$ Medical Department III for Hematology and Oncology, Klinikum rechts der Isar, Technische Universität München, Ismaninger Strasse 22, 81675 Munich, Germany

Full list of author information is available at the end of the article
}

approach is based on in vitro findings showing high efficacy of venetoclax monotherapy in high-risk MDS/sAML $[2,3]$ and synergistic effects of venetoclax and 5-AZA in primary samples [4].

Venetoclax blocks the activity of the pro-survival BCL-2 protein, priming the cells for apoptosis. Flow cytometry analysis shows an increase in BCL-2 levels and a decrease in MCL-1 levels after HMA treatment, resulting in a profile even more favorable for treatment with venetoclax (data not shown). Therefore, venetoclax treatment may reduce the apoptotic threshold in MDS or AML cells, leading to an improved response to HMA, even in cells previously resistant to HMA treatment $[4$, 5]. Interestingly, recently published data show that combination therapy of venetoclax and 5-AZA azacitidine might even eradicate leukemia stem cells by disrupting the metabolic machinery [6].

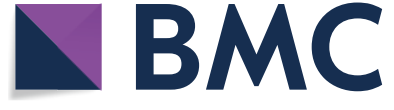

(c) The Author(s) 2019. This article is distributed under the terms of the Creative Commons Attribution 4.0 International License (http://creativecommons.org/licenses/by/4.0/), which permits unrestricted use, distribution, and reproduction in any medium, provided you give appropriate credit to the original author(s) and the source, provide a link to the Creative Commons license, and indicate if changes were made. The Creative Commons Public Domain Dedication waiver (http://creativecommons.org/ publicdomain/zero/1.0/) applies to the data made available in this article, unless otherwise stated. 
In vivo data from a phase-1b study show promising response rates of 5-AZA or decitabine in combination with venetoclax in elderly AML patients $[7,8]$ and patients with related myeloid malignancies [9].

However, a considerable concern of combination regimens in elderly AML and MDS patients is the toxicity on the remaining healthy hematopoiesis. DiNardo et al. report toxicity as manageable, yet relatively high rates of febrile neutropenia were observed [7, 8], especially in patients with relapsed/refractory disease (72\%) [9]. Furthermore, similar data were presented at ASH 2017 [10] and EHA 2018 [11] for combination therapy of venetoclax with low-dose chemotherapy.

The same safety concern was also detected in an ongoing clinical trial (Abbvie M15-531 trial), in which higherrisk MDS patients are being treated with a combination therapy of venetoclax and 5-AZA. Due to increased rate of toxic side-effects, the daily dose of venetoclax had to be reduced from 800 to $400 \mathrm{mg}$ in all arms.

Of note, as we have learned from clinical reality, cotreatment with CYP3A4 inhibitors alters venetoclax plasma concentration, maybe aggravating cytotoxic side effects [12].

Here, we report in vitro data, showing the effects of the treatment with venetoclax and 5-AZA, alone or in combination, in a cohort of MDS/sAML patients $(n=21)$ with a mean age of 72.14 (range 57-84 years), including a subset of patients resistant to HMA treatment $(n=13$, referred to as "HMA failure"), defined according to the 2006 IWG (International Working Group) response criteria (Additional file 1: Table S1) [mean age 70.08 (range 57-80 years)]. As healthy controls, we used bone marrow mononuclear cells (BMMNCs) isolated from the femoral bone of 19 elderly patients undergoing surgical hip replacement [mean age: 64.96 years (range: $49-85$ years)]. To investigate the impact on healthy hematopoiesis, we evaluated BMMNCs viability after $72 \mathrm{~h}$ treatment (shortterm) and colony forming capacity (long-term).

First, we analyzed the effects of venetoclax and 5-AZA treatment in healthy, age-matched bone marrow samples, to tailor our combination dosages towards a more tolerable regime. Primary BMMNCs were cultivated in growth-factor enriched media as previously described [2]. Viability after $72 \mathrm{~h}$-treatment was determined by flow cytometry using Annexin V and 7-amino-actinomycin D (7-AAD). To analyze colony-forming capacity, cells were pre-treated in liquid culture for $72 \mathrm{~h}$, then transferred into growth-factor enriched methylcellulose and evaluated after 10-14 days.

For all our in vitro experiments, we used venetoclax at $1 \mu \mathrm{M}$ concentration, which corresponds to the clinically used standard dose of $400 \mathrm{mg}$ [13]. In line with our previously published data $[2,3]$,
$1 \mu \mathrm{M}$ venetoclax had marginal cytotoxic effects on the bulk of BMMNCs from healthy elderly subjects (Fig. 1a). Increasing concentrations $(1-10 \mu \mathrm{M})$ of 5-AZA showed a dose-dependent toxicity for healthy bulk BMMNCs (Fig. 1a). When analyzing combination therapy of $1 \mu \mathrm{M}$ venetoclax with increasing concentrations of 5-AZA, we detected a significant decrease in cell viability (to $40.5 \%$ ). These results suggest a synergistic toxic effect of the combination therapy on the hematopoietic compartment (Fig. 1a).

To better delineate the toxic effects on hematopoiesis, we focused on the stem/progenitor compartment $\left(\mathrm{CD} 34^{+}\right)$of healthy control subjects. We found that $5 \mu \mathrm{M}, 7.5 \mu \mathrm{M}$ and $10 \mu \mathrm{M} 5$-AZA decreased the viability of $\mathrm{CD}_{34}{ }^{+}$cells by more than $50 \%$ (Fig. 1b). Cell toxicity was further increased by venetoclax, decreasing viability of the stem/progenitor compartment to below $20 \%$ (Fig. 1b).

These adverse effects were even more striking when analyzing the clonogenic potential of stem/progenitor cells in a colony-forming assay. Treatment with 5-AZA at $5 \mu \mathrm{M}, 7.5 \mu \mathrm{M}$ and $10 \mu \mathrm{M}$ significantly decreased colony numbers in healthy controls. The addition of venetoclax to 5 -AZA $(5 \mu \mathrm{M}$ or $10 \mu \mathrm{M})$ further decreased the colony forming capacity, resulting in an almost complete eradication of hematopoietic colonies (Fig. 1c). The combination therapy in vitro particularly affected the granulopoiesis, which is likely associated with the clinically-observed neutropenia (Fig. 1c). Taken together these data provide evidence of a substantial impact of venetoclax-5-AZA co-treatment on the healthy hematopoiesis. Therefore, the combination of venetoclax with 5-AZA may represent a feasible approach only when 5-AZA is capped at a dose of $1 \mu \mathrm{M}$ in order to avoid toxic side-effects.

The suggested dose for 5 -AZA is $75 \mathrm{mg} / \mathrm{m}^{2}$ of body surface, administered daily for 7 days per cycle. In daily clinical routine, $100 \mathrm{mg}$ or $200 \mathrm{mg}$ are the dosages applied. Therefore, patients with a body surface higher than $1.52 \mathrm{~m}^{2}$ receive $200 \mathrm{mg} 5$-AZA at least on 1 day of the treatment cycle. Pharmacokinetic data for doses of precisely $100 \mathrm{mg}$ and $200 \mathrm{mg}$ are not available in public databases. However, data are available for doses from $126 \mathrm{mg}$ up to $165 \mathrm{mg}$ (after subcutaneous administration) [13]. For these doses, the mean maximum concentration in the peripheral blood $\left(\mathrm{C}_{\max }\right)$ is $750 \pm 403.3 \mathrm{ng} /$ $\mathrm{ml}$, ranging from $346.7 \mathrm{ng} / \mathrm{ml}(1.42 \mu \mathrm{M})$ to $1153.3 \mathrm{ng} /$ $\mathrm{ml}(4.72 \mu \mathrm{M})[14]$.

In our in vitro setting, a combination treatment corresponding to a clinical regimen of $400 \mathrm{mg}$ venetoclax plus $100 \mathrm{mg}$ 5-AZA daily represents a lowtoxic approach. The use of higher dosages of 5-AZA as part of a combination therapy might be critically 

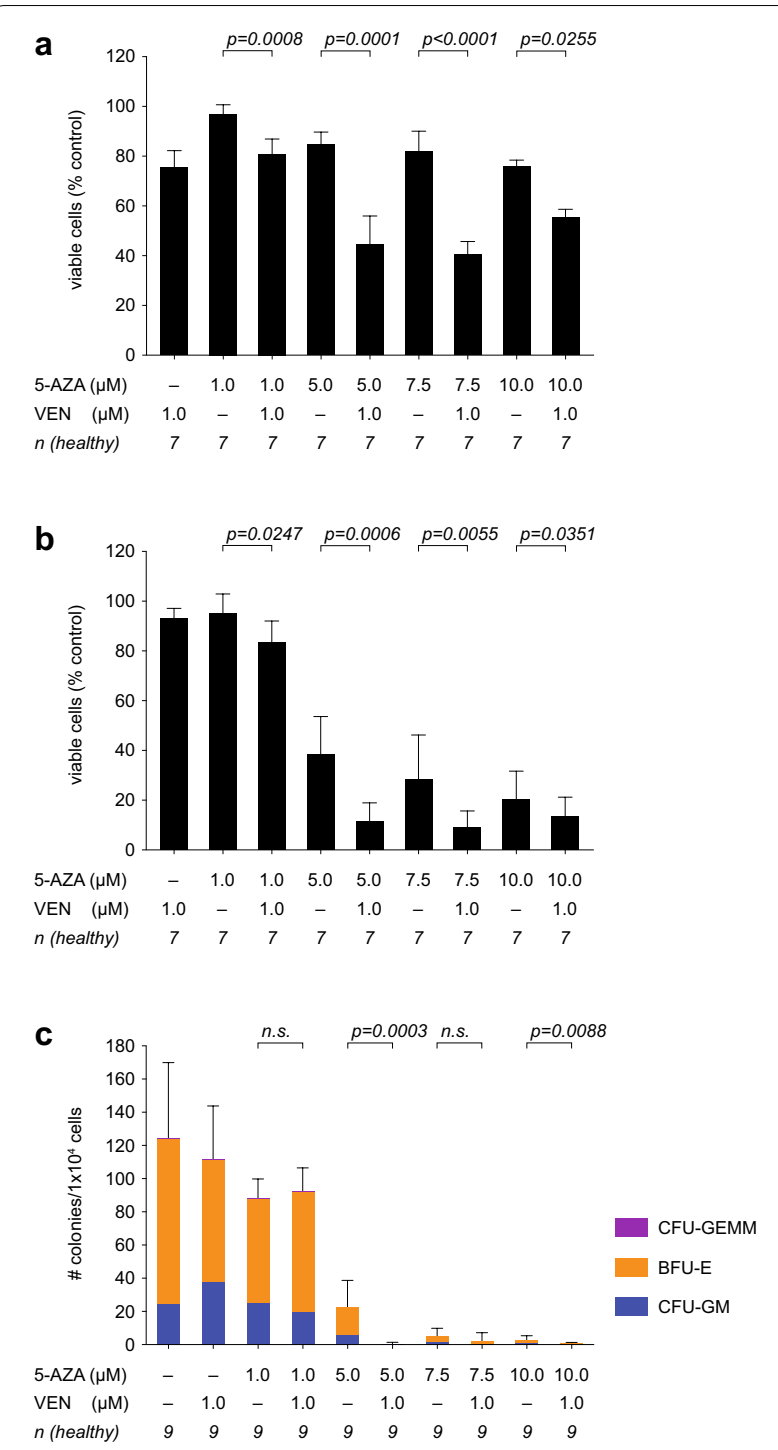

Fig. 1 Healthy hematopoiesis is affected by higher dosage combination therapy. $\mathbf{a}, \mathbf{b}$ Bone marrow mononuclear cells (BMMNCs) from healthy, elderly donors were treated for $72 \mathrm{~h}$ with the indicated concentrations of venetoclax (VEN) and 5-azacitidine (5-AZA), alone or in combination. The viability of bulk bone marrow cells $(\mathbf{a})(n=7)$ or purified CD34 ${ }^{+}$cells $(\mathbf{b})(n=7)$ was measured by flow cytometry using Annexin $\mathrm{V}$ and $7 \mathrm{AAD}$ staining. Data are presented as mean \pm standard deviation (SD) of the ratio between viable cells after a $72 \mathrm{~h}$ treatment with drug or vehicle (DMSO). One-way ANOVA resulted in $p<0.0001$ for total BMMNC (a) and $p<0.0001$ for the CD34 ${ }^{+}$compartment. Results from post hoc pairwise comparison are reported in the figure. c BMMNCs $\left(1 \times 10^{4}\right)$ from 9 individual healthy donors were plated in methylcellulose after $72 \mathrm{~h}$ of treatment with $1 \mu \mathrm{M}$ venetoclax and 5-AZA (1 $\mu \mathrm{M}, 5 \mu \mathrm{M}$, $7.5 \mu \mathrm{M}$ or $10 \mu \mathrm{M}$ as indicated), alone or in combination. The total number of colonies, distinguishing between colony-forming units (CFU) of the multi-potential granulocytic-erythroid-macrophagicmegakaryocytic lineage (CFU-GEMM), the granulocytic-macrophagic lineage (CFU-GM), and the burst-forming units-erythroid lineage (BFU-E) were determined at day 14. Experiments were performed in duplicates. One-way ANOVA resulted in $p<0.0001$. The results from post hoc pairwise comparison are reported in figure discussed in order to avoid toxic side-effects on healthy hematopoiesis.

To better elucidate the clinical relevance of the combination therapy, we tested venetoclax and 5-AZA on primary malignant MDS and sAML cells. Surprisingly, when combined with venetoclax, low-dose 5-AZA $(1 \mu \mathrm{M})$ was as effective as high-dose 5-AZA $(10 \mu \mathrm{M})$ in reducing primary malignant MDS/sAML cells (Fig. 2a). Specifically, 13 out of 19 patients (68.4\%) showed a reduction in viability below 65\% (compared to soluble control) with high-dose 5-AZA, and 5 out of 8 patients (62.5\%) using low-dose (Fig. 2a).

Patients with prior HMA failure are of special interest as treatment options for these patients remain dismal $[1,15]$. Therefore, we analysed samples from the "HMA failure" group, treated with $1 \mu \mathrm{M}$ venetoclax or $1 \mu \mathrm{M}$ 5-AZA, alone or in combination. In 11 samples, cell numbers were sufficient enough to analyse all treatments in parallel. In a small number of patients (3 out 11, 27.3\%), single-agent venetoclax showed beneficial effects (Fig. 2b). However, the combination of venetoclax with 5-AZA elicited a significant reduction in cell viability independent of IPSS grade and response to individual reagent (Fig. 2b).

To investigate the long-term effects of the combination therapy, we evaluated colony formation capacity in three individual "HMA failure" patients. In these patients, combination treatment of low-dose 5-AZA and venetoclax showed a profound effect. These results further support the notion that dose adjustments in the combination treatment will be beneficial, specifically for the high-risk group of "HMA failure" patients with MDS or sAML (Fig. 2c). To the best of our knowledge, complex karyotype or adverse mutational profile (listed in Additional file 1: Table S1) had no negative impact on treatment response (data not shown).

In summary, our data strengthen the scientific rationale of a therapeutic approach with a combination therapy using 5-AZA and venetoclax in patients with MDS/ sAML, overall in "HMA-failure" patients. These patients have an extremely poor prognosis and new therapeutic strategies are urgently needed. Interestingly, in our in vitro setting even lower-dose 5-AZA shows a valuable cytotoxicity on the malignant cell when combined with venetoclax. Toxicity on the non-malignant progenitors was significantly reduced. However, further clinical trials will be needed to test the impact of our work on clinical reality. Here, a feasible approach might be capping the daily dosage of 5-AZA at $100 \mathrm{mg} /$ day when used in a venetoclax combination regimen. This study would not only evaluate toxicity, but the effect of lower-dose 5-AZA on patients' outcome: less toxicity at the cost of efficacy may not be an acceptable trade off. 

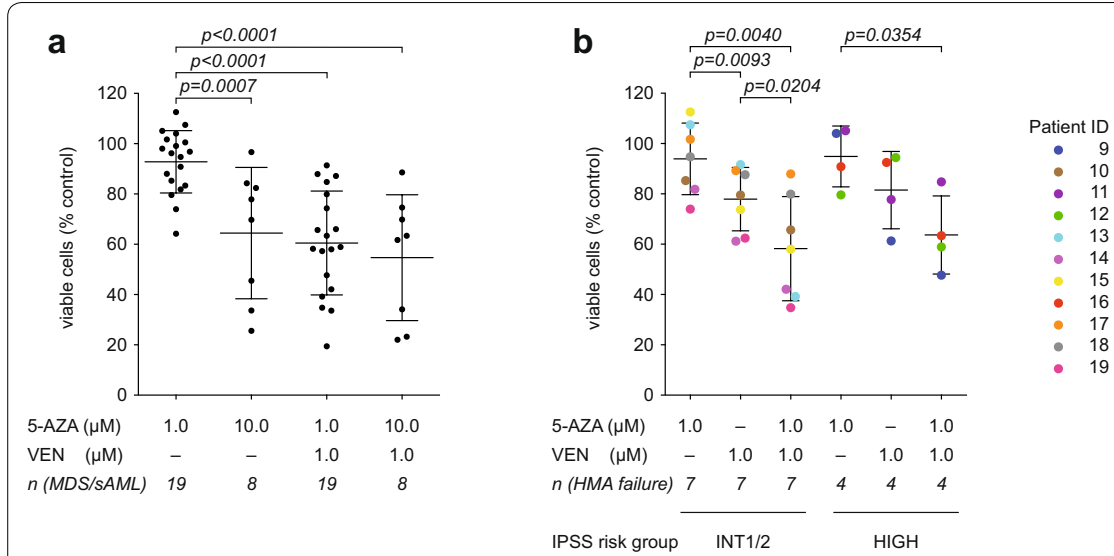

C

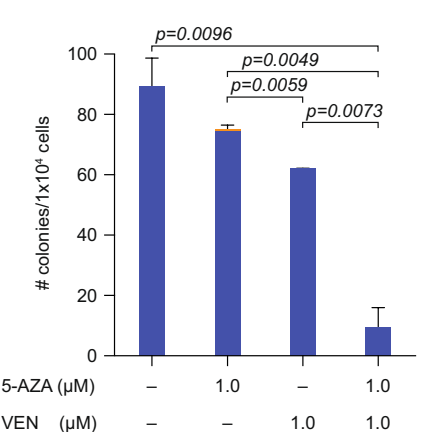

Patient \#13

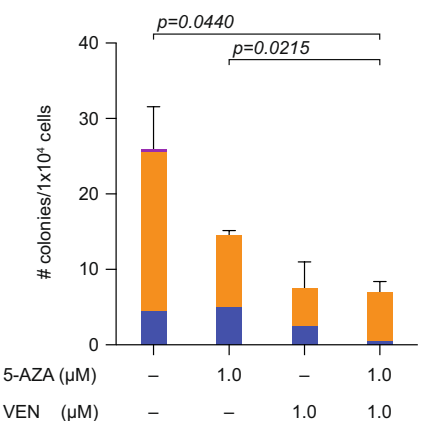

Patient \#14

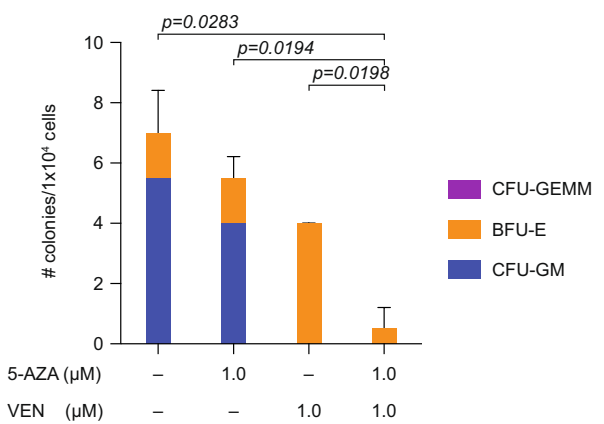

Fig. 2 Combination of 5-AZA and venetoclax is highly effective after HMA failure despite dose adjustment. a CD34+ BMMNCs from patients with Myelodysplastic Syndromes (MDS) or secondary acute myeloid leukemia (SAML) were treated for $72 \mathrm{~h}$ with venetoclax (VEN), 5-azacitidine (5-AZA), alone or in combination. Cell viability was measured by flow cytometry using Annexin $V$ and 7AAD staining. Data are presented as mean \pm standard deviation (SD) of the ratio between viable cells after a $72 \mathrm{~h}$ treatment with drug or vehicle (DMSO). One-way ANOVA resulted in $p<0.0001$. The results from post hoc pairwise comparison are reported in figure. $\mathbf{b}$ CD34 ${ }^{+}$BMMNCs from patients with MDS intermediate risk (INT) (according to IPSS) or SAML and failure of hypomethylating agent (HMA) were treated for $72 \mathrm{~h}$ with venetoclax (VEN), 5-AZA or the combination of both at the indicated concentrations. Cell viability was measured by flow cytometry using Annexin V and 7AAD staining. Data points representing the same patient are depicted in the same colour. Shown is the ratio between viable cells after a $72 \mathrm{~h}$ inhibitor or vehicle treatment (DMSO) with the mean and standard deviation (SD). One-way ANOVA resulted in $p=0.0025$. The results from post hoc pairwise comparison are reported in figure. $\mathbf{c}$ BMMNCs $\left(1 \times 10^{4}\right)$ from patient \#12, \#13 and \#14 as described in $\mathbf{b}$ with SAML or high-risk MDS (according to IPSS) after HMA failure were plated in methylcellulose after $72 \mathrm{~h}$ of treatment with Venetoclax (VEN), 5-AZA or the combination of both at the indicated concentrations. The total number of colonies was determined at day 10 to 14. Experiments were performed in duplicates. One-way ANOVA resulted in $p=0.0005$ for patient \#12, $p=0.015$ for patient $\# 13$, and $p=0.0068$ for patient $\# 14$. The results from post hoc pairwise comparison are reported in figure

\section{Additional files}

Additional file 1: Table S1. Clinical characteristics of MDS/sAML patients contributing samples. This table shows the clinical and molecular characteristics of MDS and SAML patients utilized for ex vivo treatment with venetoclax and 5-azacitidine in direct comparison.

Additional file 2. Patients and Methods.

\section{Abbreviations}

BFU-E: burst-forming units-erythroid lineage; BMMNC: bone marrow mononuclear cells; CFU: colony-forming units; CFU-GM: colony-forming units of the the granulocytic-macrophagic lineage; CFU-GEMM: colony-forming units of the multi-potential granulocytic-erythroid-macrophagic-megakaryocytic lineage; HMA: hypomethylating agents; IWG: International Working Group; MDS: myelodysplastic syndromes; sAML: secondary acute myeloid leukemia; VEN: venetoclax; 5-AZA: 5-azacitidine; 7-AAD: 7-amino-actinomycin D.

\section{Authors' contributions}

RTH performed the experiments, analyzed the data and wrote the manuscript. $J K, L B, T O O$ and VD performed the experiments. CMT,TH, PMP, BS, DH, KSG and TH provided primary samples and clinical data and gave conceptual advice. FB and CP gave conceptual advice. UH supervised the project and analyzed the data. SJ and PJJ conceived and supervised the project, analyzed the data and wrote the manuscript. All authors read and approved the final manuscript.

\section{Author details}

${ }^{1}$ Medical Department III for Hematology and Oncology, Klinikum rechts der Isar, Technische Universität München, Ismaninger Strasse 22, 81675 Munich, Germany. ${ }^{2}$ Department of Internal Medicine 3, University Hospital Grosshadern, Ludwig-Maximilians-Universität (LMU), 81377 Munich, Germany. ${ }^{3}$ Department of Orthopedic Surgery, Klinikum rechts der Isar, Technische Universität München, Munich, Germany. ${ }^{4}$ Gemeinschaftspraxis Hämato-Onkologie Pasing, Munich, Germany. ${ }^{5}$ Onkologisches Zentrum Donauwörth, Donauwörth, Germany. ${ }^{6}$ German Consortium for Translational Cancer Research (DKTK) 
of the German Cancer Research Center (DKFZ), Heidelberg, Germany. ${ }^{7}$ Munich Leukemia Laboratory (MLL), Munich, Germany.

\section{Acknowledgements}

The authors would like to thank F. Bellos and W. Worseg from MLL for providing MDS samples and editing clinical data and Caterina Branca and Amanda Kalman for giving critical advice.

\section{Competing interests}

The authors declare that they have no competing interests.

\section{Availability of data and materials}

Further information regarding Materials and methods and Clinical characteristics of MDS/SAML patients contributing samples are provided in Additional file 2.

\section{Consent for publication}

Written informed consent was obtained from each patient according to the institutional guidelines.

\section{Ethics approval and consent to participate}

Human BM samples were collected according to the institutional guidelines and in concordance with the Declaration of Helsinki. Written informed consent was obtained from each patient. The investigation was approved by the Local Ethics Committee of the University Hospital of the Technical University in Munich (Vote \#62/16S).

\section{Funding}

SJ was supported by the Hans-und-Klementia- Langmatz-Stiftung. RTH was supported by a fellowship from Deutsche José Carreras Leukämie-Stiftung/ DGHO. VD was supported by a fellowship from the DGHO/GMIHO. KSG was supported by the Deutsche José Carreras Leukämie-Stiftung (DJCLS R 14/16), the German Research Foundation (FOR 2033, Go 713/2-1 and SFB 1243 Project A09) and the Deutsche Konsortium für translationale Krebsforschung (DKTK) of the German Cancer Center (DKFZ). PJJ was supported by a Max EderProgram grant from the Deutsche Krebshilfe (program \#111738), Deutsche José Carreras Leukämie-Stiftung (DJCLS R 12/22 and DJCLS 21R/2016), Else Kröner Fresenius Stiftung (2014_A185), from the Deutsche Forschungsgemeinschaft (DFG FOR 2036) and the Deutsche Konsortium für translationale Krebsforschung (DKTK) of the German Cancer Center (DKFZ).

\section{Publisher's Note}

Springer Nature remains neutral with regard to jurisdictional claims in published maps and institutional affiliations.

Received: 18 January 2019 Accepted: 4 April 2019

Published online: 16 April 2019

\section{References}

1. Prebet T, Gore SD, Esterni B, Gardin C, Itzykson R, Thepot S, et al. Outcome of high-risk myelodysplastic syndrome after azacitidine treatment failure. J Clin Oncol. 2011;29(24):3322-7.

2. Jilg S, Reidel V, Muller-Thomas C, Konig J, Schauwecker J, Hockendorf U, et al. Blockade of $\mathrm{BCL}-2$ proteins efficiently induces apoptosis in progenitor cells of high-risk myelodysplastic syndromes patients. Leukemia. 2016;30(1):112-23.
3. Reidel V, Kauschinger J, Hauch RT, Muller-Thomas C, Nadarajah N, Burgkart $\mathrm{R}$, et al. Selective inhibition of $\mathrm{BCL}-2$ is a promising target in patients with high-risk myelodysplastic syndromes and adverse mutational profile. Oncotarget. 2018;9(25):17270-81.

4. Bogenberger JM, Kornblau SM, Pierceall WE, Lena R, Chow D, Shi $C X$, et al. BCL-2 family proteins as 5-azacytidine-sensitizing targets and determinants of response in myeloid malignancies. Leukemia. 2014;28(8):1657-65.

5. Muller-Tidow C, Schlenk RF. A new option for remission induction in acute myeloid leukaemia. Lancet Oncol. 2018;19(2):156-7.

6. Pollyea DA, Stevens BM, Jones CL, Winters A, Pei S, Minhajuddin M, et al. Venetoclax with azacitidine disrupts energy metabolism and targets leukemia stem cells in patients with acute myeloid leukemia. Nat Med. 2018;24:1859.

7. DiNardo CD, Pratz K, Pullarkat V, Jonas BA, Arellano M, Becker PS, et al. Venetoclax combined with decitabine or azacitidine in treatment-naive, elderly patients with acute myeloid leukemia. Blood. 2019;133(1):7-17. https://doi.org/10.1182/blood-2018-08-868752.

8. DiNardo CD, Pratz KW, Letai A, Jonas BA, Wei AH, Thirman M, et al. Safety and preliminary efficacy of venetoclax with decitabine or azacitidine in elderly patients with previously untreated acute myeloid leukaemia: a non-randomised, open-label, phase $1 \mathrm{~b}$ study. Lancet Oncol. 2018;19(2):216-28.

9. DiNardo CD, Rausch CR, Benton C, Kadia T, Jain N, Pemmaraju N, et al. Clinical experience with the BCL2-inhibitor venetoclax in combination therapy for relapsed and refractory acute myeloid leukemia and related myeloid malignancies. Am J Hematol. 2018;93(3):401-7.

10. Wei AH, Strickland SA, Roboz GJ, Hou J-Z, Fiedler W, Lin TL, et al. Phase 1/2 study of venetoclax with low-dose cytarabine in treatment-naive, elderly patients with acute myeloid leukemia unfit for intensive chemotherapy: 1-year outcomes (ASH abstract \#890). Blood. 2017;130:890.

11. Wei A, Tiong IS, Roberts AW, Fong CY, Chua CC, Ting SB, et al. Chemotherapy and venetoclax in elderly AML trial (CAVEAT): a phase 1B dose escalation study examining modified intensive chemotherapy in fit elderly patients (EHA abstract \#S1564). HemaSphere. 2018;2(S1).

12. Agarwal SK, Salem AH, Danilov AV, Hu B, Puvvada S, Gutierrez M, et al. Effect of ketoconazole, a strong CYP3A inhibitor, on the pharmacokinetics of venetoclax, a BCL-2 inhibitor, in patients with non-Hodgkin lymphoma. Br J Clin Pharmacol. 2017;83(4):846-54.

13. Roberts AW, Davids MS, Pagel JM, Kahl BS, Puvvada SD, Gerecitano JF, et al. Targeting BCL2 with venetoclax in relapsed chronic lymphocytic leukemia. N Engl J Med. 2016;374(4):311-22.

14. Marcucci G, Silverman L, Eller M, Lintz L, Beach CL. Bioavailability of azacitidine subcutaneous versus intravenous in patients with the myelodysplastic syndromes. J Clin Pharmacol. 2005;45(5):597-602.

15. Jabbour E, Garcia-Manero G, Batty N, Shan J, O'Brien S, Cortes J, et al. Outcome of patients with myelodysplastic syndrome after failure of decitabine therapy. Cancer. 2010;116(16):3830-4.

Ready to submit your research? Choose BMC and benefit from:

- fast, convenient online submission

- thorough peer review by experienced researchers in your field

- rapid publication on acceptance

- support for research data, including large and complex data types

- gold Open Access which fosters wider collaboration and increased citations

- maximum visibility for your research: over $100 \mathrm{M}$ website views per year

At BMC, research is always in progress.

Learn more biomedcentral.com/submissions 